\title{
Paciente joven con disfonía: un caso de amiloidosis poco habitual
}

\author{
Alicia Maria Aquino Valdovinos', Maria Isabel Acosta Colmán', Hugo Boggino*, Margarita Duarte \\ Mussi de Laterza ' \\ I. Departamento de Reumatología del Hospital de Clínicas, Facultad de Ciencias Médicas, \\ Universidad Nacional de Asunción
}

Cómo referenciar este artículo/ How to reference this article:
Aquino A, Acosta I, Boggino H, Duarte M. Paciente joven con disfonia: un caso de amiloidosis poco habitual. Mem. Inst. Investig. Cienc. Salud. 2015; 13(2): 103-7

\section{R E S U M E N}

La amiloidosis es una enfermedad caracterizada por el depósito extracelular de fibrillas compuestas por subunidades de bajo peso molecular de una variedad de proteínas. Puede ser clasificada según su distribución y según la proteina fibrilar constituyente. La prevalencia varía según el área geográfica estudiada y el pronóstico depende del tejido u órgano afectado. Presentamos el caso de un varón jóven con disfonía de larga evolución sin otros síntomas acompañantes en cuya nasofibroscopía se observaron una tumoración en regíon laríngea. En la anatomía patológica se observó material hialino congofílico compatible con amiloide. Se realizó además una biopsia rectal donde también se observó material amiloide. Debido a la poca sintomatología y a la ausencia de compromiso de un órgano que comprometa la vida, en este paciente se optó por un manejo conservador.

Palabras clave: amiloidosis sistémica, disfonía, laringe.

\section{Young patient with dysphonia: a case of unusual Amyloidosis}

\section{A B S T R A C T}

Amyloidosis is a disease characterized by extracellular deposition of fibrils made of low molecular weight subunits of a variety of proteins. It can be classified according to its distribution and the fibrillar protein constituent. The prevalence varies by the geographical area studied and the prognosis depends on the tissue or organ concerned. We present the case of a young man with longstanding dysphonia without other accompanying symptoms whose nasofibroscopy showed a tumor in the laryngeal region. In the pathological material, hyaline congophillic material compatible with amyloid was observed. A rectal biopsy was also performed and amyloid material was also observed. Due to the few symptoms and the absence of organ involvement that compromises life, we opted for a conservative management in this patient.

Keywords: systemic amyloidosis, dysphonia IDEM, larynx

\section{NTRODUCCI ON}

La amiloidosis es una enfermedad que se caracteriza por el depósito extracelular de fibrillas compuestas por subunidades de bajo peso molecular de una variedad de proteínas. Esta enfermedad fue descrita por primera vez por Virchow quien en 1.854 pensó que los depósitos se asemejaban a la celulosa (1).

Existen varias clasificaciones con respecto a la amiloidosis, que permiten clasificarla de acuerdo a su distribución y la proteína fibrilar constituyente; una de ellas es la propuesta en 1.993 por la WHO-IUIS (World Health Organization/International Union of 
Immunological Societies) que tiene en cuenta la etiopatogenia de la enfermedad. Dicha clasificación reconoce seis tipos de amiloidosis, la amiloidosis primaria ( $A L)$, debida al depósito de cadenas ligeras de inmunoglobulinas, la amiloidosis secundaria (AA) o secundaria a enfermedades crónicas, la amiloidosis asociadas a diálisis debida al depósito de B2 microgobulina, la amiloidosis hereditaria o por depósito de fibrillas de transtiretina (prealbumina), la amiloidosis asociada con la edad y finalmente la amiloidosis localizada $(2,3)$.

La prevalencia de los subtipos de amiloidosis varía según el grupo poblacional estudiado. En los países en vías en desarrollo, predomina la amiloidosis secundaria debido a la alta prevalencia de enfermedades crónicas (por ejemplo: las infecciones por micobacterias), al contrario de los países desarrollados donde predomina la amiloidosis primaria (4).

Una de las manifestaciones clínicas de la amiloidosis es la afectación renal en forma de proteinuria, hipertensión arterial y síndrome nefrótico. Además puede afectar otros aparatos y sistemas produciendo hepatomegalia, macroglosia, síndrome del túnel carpiano, miocardiopatía restrictiva, neuropatía periférica sensitivo motora, diarrea y/o malabsorción, artropatía por infiltración sinovial (5-9).

El diagnóstico de la amiloidosis se realiza fundamentalmente por biopsia del órgano disfuncional o de la grasa subcutánea, de las glándulas salivales o de la mucosa rectal. Como dijimos anteriormente, debido a la heterogenicidad de la enfermedad y por sobre todo a la poca frecuencia de la misma, el diagnóstico de la amiloidosis muchas veces se ve retrasado. Por todo esto, en este artículo presentamos el caso clínico de un paciente con amiloidosis con una presentación clínica poco habitual.

\section{CASO CLI NI CO}

Paciente, varón de 29 años de edad que acudió al consultorio externo del departamento de Reumatología del Hospital de Clínicas de la Facultad de Medicina de la Universidad Nacional de Asunción por disfonía. El paciente no tenía historia de patología de base hasta que hace dos años presentó una disfonía de inicio insidioso y de instalación progresiva sobre todo en los meses previos a la consulta.

Al interrogatorio funcional el paciente negó historia de disfagia, disnea, síntomas constitucionales, fiebre, artralgias. Tampoco se registraron antecedentes familiares de patologías oncológicas o autoinmunes ni consumo de sustancias ilícitas.

Al examen físico se constató un ritmo cardiaco regular, no se auscultaron soplos, murmullo vesicular conservado; no se objetivó organomegalias y las articulaciones no presentaron sinovitis ni deformidades.

En los exámenes laboratoriales realizados no se constataron anormalidades llamativas tal y como se observa en la Tabla 1.

Se realizó una ecografía renal, una ecocardiografía, una radiografía de tórax y una tomografía cervical sin datos de valor. En la nasofibroscopía se observó una tumoración en la glotis por lo cual se realizó exéresis de dicha tumoración. En la anatomía patológica de la pieza anatómica se observó una gran cantidad de material amorfo hialino depositado en mucosa respiratoria y en las paredes de los vasos sanguíneos; congofílico y birrefringente a la luz polarizada compatible con material amiloide. Frente a este hallazgo, se realizó una biopsia de la submucosa rectal que también fue positiva para material amiloide.

Finalmente, se realizó punción de médula ósea, electroforesis de proteínas y dosaje de cadenas livianas libres kappa y lambda que resultaron normales, descartándose gammapatías monoclonales. 
Tabla 1. Estudios laboratoriales realizados

\begin{tabular}{lll}
\hline Estudios laboratoriales & Resultados & Valores de referencia \\
\hline Hemoglobina (g/dL) & 15,4 & $14-16$ \\
Hematocrito (\%) & 40,1 & $36-45$ \\
Glóbulos blancos (cel/ $\mathrm{mm}^{3}$ ) & 6.500 & $4.000-11.000$ \\
Neutrófilos (\%) & 44 & $45-65$ \\
Linfocitos (\%) & 45 & $15-45$ \\
Ácido úrico (mg/dL) & 7,1 & $3,5-7,2$ \\
GOT (U/L) & 32 & $5-33$ \\
GPT (U/L) & 51 & $10-40$ \\
Urea (mg/dL) & 58 & $10-50$ \\
Creatinina (mg/dL) & 1,3 & $0,8-1,5$ \\
Albumina (g/dL) & 4,4 & $3,5-5$ \\
Orina simple & Leucocitos 0-1, Hematíes 1-3 & $<10$ cel/campo, 1-5 cel/campo \\
Factor Reumatoide & Negativo & Negativo \\
ANA & Negativo & Negativo \\
Anti DNA & Negativo & Negativo \\
Eritrosedimentación (mm) & 8 en la 10 hora & Hasta 20 \\
PCR (mg/L) & $<6$ & $<6$ \\
\hline
\end{tabular}

La opción terapéutica a realizar podría ser la extirpación quirúrgica de los nódulos laríngeos ya que es la única manifestación de la enfermedad hasta el momento pero como el paciente se mantiene estable e inclusive con una ligera mejoría de la disfonía posterior a la toma de biopsia de glotis se optó por un manejo expectante y conservador.

\section{SCUSIÓN}

La amiloidosis es una enfermedad relativamente rara, en la cual las formas sistémicas - localizadas tienen diferentes pronósticos por lo que requieren diferentes regímenes de tratamiento. Hemos presentado el caso de un paciente con amiloidosis sistémica en un paciente de sexo masculino de 29 años de edad, con disfonía secundaria a una afectación laríngea como manifestación clínica inicial.

La amiloidosis es una enfermedad en la cual se dificulta la obtención de datos fiables sobre la incidencia y prevalencia de la misma ya que suele ser erróneamente diagnosticada al inicio de la enfermedad. La prevalencia de diferentes subtipos de amiloidosis varía según la localización geográfica estudiada, así en los países subdesarrollados predomina la amiloidosis secundaria mientras que en los países desarrollados predomina la primaria. En Paraguay, no se cuenta con estudios epidemiológicos sobre esta enfermedad por lo que se desconoce la prevalencia e incidencia de esta patología (5).

Como comentamos anteriormente la amiloidosis puede ser sistémica o localizada. Las tres formas de amiloidosis sistémica incluyen la forma primaria, la forma reactiva o secundaria y la forma familiar. La forma localizada es una variedad poco frecuente. No obstante, la amiloidosis en ocasiones puede ser clasificada inicialmente como localizada, para posteriormente presentarse como sistémica por lo que es necesario realizar un seguimiento adecuado para descartar la presencia de depósitos de amiloide en otros órganos.

La afectación laríngea como la que presentó este paciente, puede ser observada tanto 
en las amiloidosis sistémicas como las localizadas. En relación a la amiloidosis localizada la afectación laríngea (por ejemplo: cuerdas vocales) es considerada una de las afectaciones más frecuentes en cuanto a compromiso del tracto respiratorio superior $(10,11)$. No obstante en la amiloidosis sistémica, como la observada en este paciente, la afectación laríngea como primera manifestación de la enfermedad es reportada en la literatura en casos aislados (12).

Lewis et al. (13) estudiaron 22 pacientes de la Clínica Mayo, de Rochester, EEUU; que presentaron el diagnóstico de amiloidosis localizada en la laringe durante un periodo de 1.950 y 1.988 . En un trabajo realizado por Thompson et al., en la mayoría de los casos que se diagnosticaron como amiloidosis primaria focal resultaban amiloidosis primarias sistémicas años más tarde (14). En nuestro paciente la afectación laríngea es la primera manifestación de una amiloidosis sistémica, ya que se constató afectación en la mucosa rectal como comentamos anteriormente.

El diagnóstico de amiloidosis se realiza por electroforesis sérica y urinaria y para casos específicos, por medio de la biopsia del intestino, del tejido celular subcutáneo o biopsia de médula ósea o de localizaciones específicas. En este paciente el diagnóstico se realizó por medio del hallazgo de depósito amiloide en las cuerdas vocales y posteriormente en mucosa rectal que fue positivo para la refringencia en luz polarizada con la tinción con rojo congo; dicha tinción es la más comúnmente utilizada para detectar la proteína amiloide en los tejidos estudiados (15-17).

El tratamiento de la amiloidosis varía de acuerdo a la causa de la producción de las fibrillas. Por ejemplo, la terapia está dirigida a una infección subyacente o un desorden inflamatorio en la amiloidosis secundaria, tratamiento de la discrasia de células plasmáticas en el caso de la amiloidosis primaria, o a cambiar el modo de diálisis e inclusive trasplante renal en pacientes con amiloidosis secundaria a diálisis. En el caso de nuestro paciente debido a la sintomatología localizada, la opción terapéutica sería la quirúrgica pudiendo realizarse inclusive la exéresis con láser (18-22), pero debido a la mejoría posterior a la toma de biopsia de glotis se optó por un tratamiento conservador.

En este artículo se presenta un caso de amiloidosis sistémica con afectación laríngea que fue responsable de la disfonía como primera manifestación clínica de la enfermedad. Considerando la baja frecuencia de esta patología y sobre todo las diferentes formas de presentación clínica inicial, es importante conocer esta patología a fin de considerar la amiloidosis dentro del algoritmo diagnóstico ya que para su confirmación solo llegamos a ella con la biopsia y las técnicas correctas para esto, permitiendo así un diagnóstico precoz y un seguimiento adecuado para detectar las complicaciones que requieran una intervención terapéutica.

\section{REFERENCI AS BIBLI OGRÁFI CAS}

1. Sipe JD, Benson MD, Buxbaum JN, Ikeda S, Merlini G, Saraiva $M J$, et al. Amyloid fibril protein nomenclature: 2012 recommendations from the Nomenclature Committee of the International Society of Amyloidosis. Amyloid. 2012 Dec; 19(4): 16770.

2. Westermark $P$, Benson MD, Buxbaum JN, Cohen AS, Frangione B, I keda $S$, et al. A primer of amyloid nomenclature. Amyloid. 2007 Sep; 14(3): 179-83.

3. Nomenclature of amyloid and amyloidosis. WHO-IUIS Nomenclature Sub-Committee. Bull World Health Organ 1993; 71(1): 105-12.
4. Kyle RA, Linos A, Beard CM, Linke RP, Gertz MA, O'Fallon WM, Kurland LT. Incidence and natural history of primary systemic amyloidosis in Olmsted County, Minnesota, 1950 through 1989. Blood. 1992;79(7): 181722.

5. Merlini G, Palladini G. Amyloidosis: Is a cure possible?. Ann Oncol. 2008; 19(4):63-6.

6. Kurita N, Kotera N, Ishimoto Y, Tanaka M, Tanaka S, Toda N, et al. AA amyloid nephropathy with predominant vascular deposition in Crohn's disease. Clin Nephrol. 2013; 79(3): 229-32.

7. Shin SC, Robinson-Papp J. Amyloid neuropathies. Mt Sinai J Med. 2012;79(6):733 -48 . 
8. Sattianayagam $P$, Hawkins $P$, Gillmore J. Amyloid and the Gl tract.1. Expert Rev Gastroenterol Hepatol. 2009; 3(6):615-30.

9. Yood RA, Skinner $M$, Rubinow A, Talarico $L$, Cohen AS. Bleeding manifestations in 100 patients with amyloidosis. JAMA. 1983;249 (10): 1322-4.

10. Vázquez de la Iglesia F, Sánchez Ferrándis N, Rey Martínez J, Ruba San Miguel D, Rama López J, Fernández González S. Amyloidosis in the ORL field. Acta Otorrinolaringol Esp. 2006; 57(3): 145-8.

11. Raymond AK, Sneige N, Batsakis JG. Amyloidosis in the upper aerodigestive tracts. Ann Otol Rhinol Laryngol. 1992;101(9):7946.

12. Thompson LD, Derringe GA, Wenig BM. Amyloidosis of the Larynx: A clinico pathologic study of 11 cases. Mod Pathol. 2000;13 (5): 528- 35 .

13. Lewis JE, Olsen KD, Kurtin PJ, Kyle RA. Laryngeal amyloidosis: A clinico pathologic and immunohistochemical review. Otolaryngol Head Neck Surg. 1992; 106( 4):372-7.

14. Yiotakis I, Georgolios A, Charalabopoulos A, Hatzipantelis P, Golias C, Charalabopoulos K, Manolopoulos L. Primary localized laryngeal amyloidosis presenting with hoarseness and dysphagia: A case report. Journal of Medical Case Report. 2009; 3:9049

15. Westermark P, Davey E, Lindbom K, Enqvist S. Subcutaneous fat tissue for diagnosis and studies of systemic amyloidosis. Acta Histochem. 2006; 108(3): 209-13.

16. Van Gameren II, Hazenberg BP, Bijzet J, van Rijswijk $\mathrm{MH}$. Diagnostic accuracy of subcutaneous abdominal fat tissue aspiration for detecting systemic amyloidosis and its utility in clinical practice. Arthritis Rheum. 2006; 54(6) : 2015-21.
17.Dhingra S, Krishnani N, Kumari N, Pandey R. Evaluation of abdominal fat pad aspiration cytology and grading for detection in systemic amyloidosis. Acta Cytol. 2007; 51(6): 860-4.

18. Herlenius G, Wilczek HE, Larsson M, Ericzon BG, Familial Amyloidotic Polyneuropathy World Transplant Registry. Ten years of international experience with liver transplantation for familial amyloidotic polyneuropathy: results from the Familial Amyloidotic Polyneuropathy World Transplant Registry. Transplantation. 2004; 77(1):64-71.

19.Tsuchiya A, Yazaki M, Kametani F, Takei $Y$, Ikeda S. Marked regression of abdominal fat amyloid in patients with familial amyloid polyneuropathy during long-term follow-up after liver transplantation. Liver Transpl. 2008; 14(4): 563-70.

20.Benson MD. Liver transplantation and transthyretin amyloidosis. Muscle Nerve 2013; 47 (2): 157-62.

21. Yamamoto S, Wilczek HE, Nowak G, Larsson M, Oksanen A, Iwata $T$, et al. Liver transplantation for familial amyloidotic polyneuropathy (FAP): Asingle-center experience over 16 years. Am J Transplant. 2007; 7(11): 2597-604.

22. Nelson LM, Penninga L, Sander K, Hansen PB, Villadsen GE, Rasmussen A, Gustafsson F. Long-term outcome in patients treated with combined heart and liver transplantation for familial amyloidotic cardiomyopathy. Clin Transplant. 2013;27 (2): 203-9. 